Polo Sánchez, M.T. y Aparicio Puerta, M. (2018). Primeros pasos hacia la inclusión: Actitudes hacia la discapacidad de docentes en educación infantil. Revista de Investigación Educativa, 36(2), 365-379.

DOI: http://dx.doi.org/10.6018/rie.36.2.279281

\title{
Primeros pasos hacia la inclusión:Actitudes hacia la discapacidad de docentes en educación infantil
}

\section{First steps towards inclusion:Attitudes towards disability in early childhood education teachers}

\author{
M. Tamara Polo Sánchez y Marta Aparicio Puerta \\ Departamento de Psicología Evolutiva y de la Educación. Facultad de Ciencias de la Educación. \\ Universidad de Granada (España)
}

\begin{abstract}
Resumen
Se considera que el medio más eficaz para crear una sociedad más justa e igualitaria es una escuela inclusiva, que garantice a todos los niños, independientemente de sus características y necesidades educativas, el derecho a una educación de calidad (UNESCO, 1994). Uno de los elementos esenciales a la hora de llevar a cabo una inclusión educativa es la actitud de los docentes. Por ello, el objetivo de este estudio es analizar las actitudes que tienen los docentes de educación infantil de Granada acerca de los estudiantes con necesidades educativas especiales. Para ello, se aplicó a 78 docentes de Educación Infantil, de 14 centros la provincia de Granada, la Escala de Actitudes hacia las personas con discapacidad (Verdugo, Jenaro E Arias, 2002). De los resultados obtenidos se puede concluir que existe, en general, una actitud positiva hacia la discapacidad, siendo los docentes de segundo ciclo de Educación Infantil los que presentan actitudes más positivas. Se discuten los resultados, y se concluye como nos encontramos ante un estudio pionero en su campo, ya que no se han encontrado investigaciones que aborden ambos ciclos de Educación Infantil. Esto permite analizar si los docentes de esta etapa tienen la formación necesaria para llevar a cabo una educación de calidad para
\end{abstract}

Correspondencia: M. Tamara Polo Sánchez, tpolo@ugr.es. Psicología Evolutiva y de la Educación, Facultad de Ciencias de la Educación, Universidad de Granada, Campus Universitario Cartuja, s/n, 18071, Granada. España. 
todo el alumnado. La necesidad de centrarnos en esta etapa radica en el carácter preventivo de la misma, en la medida en que la inclusión educativa como prevención para la exclusión social es el medio más efectivo para combatir las actitudes de discriminación (Echeita, 2006).

Palabras clave: actitudes; discapacidad; diversidad; educación de la primera infancia; educación superior; profesorado.

\section{Abstract}

It is considered that the most effective way to create a more just and egalitarian society is an inclusive school that guarantees all children, regardless of their characteristics and educational needs, the right to quality education (UNESCO, 1994). One of the essential elements when conducting an educational inclusion is the attitude of teachers. Therefore, the aim of this study is to analyze the early childhood education teachers' attitudes toward students with special educational needs. To this end, it was applied the scale ot attitudes towards disabled people to 78 early childhood education teachers, from 14 centers in the province of Granada (Verdugo, Jenaro\& Arias, 2002). From the results it can be concluded that there is generally a positive attitude towards disability, being teachers of second cycle of early childhood education those with more positive attitudes. The results are discussed, and we conclude that we offer a pioneering study in its field, since no research has been found that addresses both cycles of Early Childhood Education. This allows to analyze if the teachers of this stage have the necessary training to carry out a quality education for all the students. The need to focus on this stage lies in its preventive nature, because the inclusive education as prevention for social exclusion is the most effective means of combating attitudes of discrimination (Echeita, 2006).

Keywords: attitudes; disability; diversity; early childhood education; higher education; teachers.

\section{Introducción}

Las personas con discapacidad se caracterizan por ser uno de los grupos sociales más marginados. Apenas participan en la economía, disponen de menores beneficios en los servicios sanitarios, registran resultados académicos inferiores al resto de sus compañeros e incluso, poseen una tasa de pobreza superior al resto de personas (Organización Mundial de la Salud, 2011). De esta forma, se considera que el medio más eficaz para crear una sociedad más justa e igualitaria es una escuela inclusiva, que garantice a todos los niños, independientemente de sus características y necesidades educativas, el derecho a una educación de calidad (UNESCO, 1994). Según Cortés (2010), son numerosas las ventajas que posee la inclusión en el ámbito educativo. Así por ejemplo, mejora el desarrollo emocional del niño con necesidades educativas especiales (NEE) pues al estar integrado en el aula, no se siente diferente a los demás y por otro lado, aumenta la aceptación del resto de sus compañeros. Al aumentar su autoestima y su pertenencia a un grupo, también se verá mejorado su rendimiento académico. De la misma forma, es muy probable que esta inclusión influya de forma favorable en el rendimiento escolar del resto de alumnos. La inclusión social de las personas con discapacidad se viene tratando desde el marco político y legal español, que está generando políticas y normas de actuación orientadas a minimizar o 
suprimir algunas barreras.También es el caso de las medidas relativas a mejorar la infraestructura, los espacios y la supresión de barreras arquitectónicas de acceso. Sin embargo, a pesar de los intentos, por mejorar las condiciones y expectativas de los niños con discapacidad que desde distintas instancias se vienen haciendo, todavía importantes hitos de la vida adulta como el empleo, la vida independiente o la inclusión en la comunidad, permanecen fuera del alcance de muchos de éstos jóvenes (Peralta, 2011). En este sentido, es fundamental un cambio en las creencias, valores y cultura de los profesionales implicados, a favor de una visión más positiva y comprensiva de la discapacidad. Existen otras barreras más sutiles para la inclusión de las personas con discapacidad, sobre las que se requiere trabajar: las barreras actitudinales. Se entiende por actitud, un conjunto de creencias y sentimientos que condicionan y orientan nuestro comportamiento (Noe, 2002). Escámez, García, Pérez y Llopis (2007) aseguran que estas actitudes son cambiantes y educables. Por tanto, uno de los factores más importantes a tener en cuenta a la hora de llevar a cabo una inclusión educativa con éxito es la actitud favorable que han de tener todos los agentes implicados en este proceso. Rodríguez, Etopa y Rodríguez (2002), aseguran que en el terreno de las actitudes hacia la discapacidad, es fundamental el papel del docente. Estos, a través de sus percepciones y actitudes pueden influir en el aprendizaje de sus alumnos, bien de forma directa o a través de su autoestima y motivación (Sales, Moliner \& Sanchiz-Ruiz, 2001). Por tanto, para que la inclusión escolar se lleve a cabo de una manera eficaz y satisfactoria, no es suficiente la implantación de leyes, sino que además, son fundamentales las actitudes que los docentes tienen hacia la discapacidad, ya que estas pueden llegar a ser una de las barreras más importantes para los alumnos. Cobra especial interés en la etapa de educación infantil, dado su marcado carácter preventivo (Ministerio de Educación, Cultura y Deporte, 2001) indagar en las actitudes de los docentes, pues son los primeros en tener contacto con el niño al inicio de la escolarización. En una revisión sobre las actitudes hacia la discapacidad, León (1995) señaló las variables que han correlacionado con la actitud de los profesores hacia la inclusión. Entre ellas destacó las variables de tipo estáticas como el género, la edad; los años de experiencia docente, el contacto con personas con discapacidad, y el nivel educativo-formación del docente.

En lo que respecta al género de los docentes, los resultados obtenidos por diferentes autores han sido inconsistentes. Mientras que algunos estudios afirman que las profesoras tienen una mayor tolerancia hacia la inclusión (Eichinger, Rizzo \& Sirotnik, 1991), otros no han encontrado diferencias en función de esta variable (Galović, Brojčin \& Glumbić, 2015; García \& Alonso, 1985; Parasuram, 2006; Sánchez, 2011). En cambio, Abós y Polaino (1986), destacaron que una de las variables que influyen negativamente es el género, siendo los hombres quienes poseen una actitud más favorable a la integración. De la misma forma, la edad y los años de experiencia docente también parecen tener efecto, siendo los más jóvenes (Dengra, Durán \& Verdugo, 1991; García \& Alonso, 1985) y con menos de cinco años de experiencia los que presentaban actitudes más positivas (Parasuram, 2006). Con respecto al contacto, son varios los autores (Bilbao, 2008; Díaz, 2004; Martínez \& Bilbao, 2011) que afirman que aquellos profesionales que han tenido contacto con alumnos con discapacidad se sienten más capaces, y conceden mayores posibilidades 
de integración. No obstante, cuando se analiza la influencia sobre las actitudes de la frecuencia del contacto los resultados no son concluyentes (García \& Hernández, 2011; Gómez \& Infante, 2004; López-Ramos, 2004; Moreno, Rodríguez, Saldaña \& Aguilera, 2006; Muratori, Guntín \& Delfino, 2010; Parasuram, 2006; Yazbeck, Mcvilly \& Parmenter, 2004).

Numerosas investigaciones, como las llevadas a cabo por Carberry, Waxman y McKain (1981), Tenorio (2011) y Verdugo, Jenaro y Arias (2002) resaltan la importancia de la formación del profesorado a la hora de mejorar las actitudes hacia las personas con discapacidad. Esta misma idea se ve apoyada por las investigaciones de Ashman (1982) y Sánchez y Carrión (2002), quienes concluyen que es la falta de formación por parte del profesorado lo que les impide llevar a cabo su labor educativa de una forma adecuada, y por tanto, la formación permanente en este ámbito, les permite obtener una mayor seguridad en sí mismos a la hora de trabajar con alumnos con NEE, lo que se traduce en una mejor enseñanza. Por su lado, Clark (1976), Harasymiw y Horme (1976) y Toledo (1984), en sus estudios destacan que además de la formación, también existe otra variable determinante en el éxito de la enseñanza con alumnos con NEE, como la presencia de personal especialista en el aula. En un estudio llevado a cabo por Doménech, Esbrí, González y Miret (2004), se concluye que una actitud más negativa hacia la discapacidad está vinculada con una falta o escasa formación en el tema. La formación del profesorado ha sido identificado como un principal contribuyente a las actitudes del maestro (Stauble, 2009). En esta vía, un amplio campo de estudio lo ha constituido el análisis de las actitudes de docentes y futuros docentes en distintos niveles educativos. En general, aunque no se han encontrado diferencias significativas en las actitudes de los profesores en función de la etapa educativa (Chiner, 2011), Galović et al. (2015) en un estudio en el que analizaron las actitudes hacia la inclusión de alumnos con NEE, de los docentes de educación infantil, primaria, secundaria y universidad, encontraron que los docentes de universidad y educación infantil, con experiencias previas en ambientes inclusivos, informaban de actitudes más positivas que los de primaria y secundaria. Son numerosos los estudios que demuestran en el ámbito universitario una actitud positiva por parte de los docentes hacia los alumnos con discapacidad (Comes, Parera, Vedriel \& Vives, 2011; Garabal-Barbeira, 2015; Mayo, 2012; Polo, Fernández \& Fernández, 2017; Rodríguez-Martín \& Álvarez-Arregui, 2015; Sánchez, 2011; Soto, 2007). De la misma forma, otros autores, como Álvarez, Castro, CampoMon y Álvarez-Martino (2005) se centraron en etapas inferiores, resaltando en un estudio llevado a cabo con una amplia muestra de maestros de Educación Primaria, una actitud positiva por parte de los docentes hacia el alumnado con discapacidad. La misma actitud positiva se refleja de forma general en otra investigación llevada a cabo por Doménech et al. (2004) con docentes de Educación Infantil, Primaria y Secundaria. Sin embargo, son escasas las investigaciones realizadas en la etapa de Educación Infantil. En un estudio realizado por Santiago y Marrero (2003), con una muestra de 36 educadores, concluye que estas profesionales tienen una actitud positiva hacia la discapacidad. No obstante, no se analiza la influencia de variables como la edad o la formación. Asimismo, Gal, Schreur y Engel-Yeger (2010), tras llevar a 
cabo una investigación con 53 maestros y maestras de Educación Infantil, resaltaron la edad y los años de experiencia como factores determinantes en la actitud de los docentes hacia la discapacidad, concluyendo que existe una relación negativa entre estas dos variables y una actitud positiva ante este alumnado.

En un reciente trabajo Kwon, Hong y Jeon (2017), han examinado, la relación entre las experiencias de los maestros con respecto a los niños con discapacidad (es decir, la educación, la formación especializada, los años de experiencia laboral), y sus actitudes hacia los mismos. La formación previa y específica del docente, así como el contacto con personas con discapacidad se relacionaría con actitudes positivas.

Bajo estas premisas, todo ello justifica que se plantee el presente estudio, cuyo cometido es en primer lugar analizar las actitudes que tienen los docentes en infantil acerca de los estudiantes con NEE, y en segundo lugar, analizar la influencia que ejerce sobre dichas actitudes variables tales como género, edad, años de experiencia docente, contacto con personas discapacitadas, y nivel educativo-formación del docente.

\section{Método}

\section{Participantes}

Para seleccionar los centros participantes en esta investigación, se llevó a cabo un muestreo intencional. Algunos de los criterios que se tuvieron en cuenta a la hora de la selección fueron: el tipo de colegio (público, privado o concertado), la localización del mismo y las características de los maestros que imparten docencia en él. El muestreo intencional constituye una estrategia no probabilística especialmente útil para muestras pequeñas y muy específicas (Alaminos, 2006). Como afirma Quinn (1980), la importancia del muestreo estadístico radica en seleccionar una muestra realmente aleatoria y representativa que permita hacer generalizaciones desde la muestra seleccionada una población mayor. Lo fundamental en el muestreo intencional es seleccionar casos ricos en información para poder estudiarlos en profundidad. De esta forma, se consiguió la participación de 78 maestros de Educación Infantil, que impartían docencia en 14 centros diferentes de la provincia de Granada durante el curso 2015-2016, tanto públicos, privados o concertados, como de primer (0-3 años) o segundo ciclo (3-6 años).

En la tabla 1 se pueden visualizar los datos más representativos relativos a la muestra del profesorado participante. Así, se observa como la mayor parte de la muestra está conformada por mujeres (91\%), de entre $41-50$ años (33\%), con estudios universitarios de grado medio $(55.7 \%)$.

En la tabla 2 se muestran las características de los centros donde los maestros, maestras, educadores y educadoras imparten docencia. Se puede observar que la mayoría son centros públicos $(39.8 \%)$ y concertados $(41 \%)$, localizados en general, en Granada (64.1\%). Igualmente, una gran parte de los entrevistados imparte docencia en el segundo ciclo de Educación Infantil (64.1\%), y el restante 35.9\% en primer ciclo. Además, el 17.9\% de los participantes tiene menos de 20 años de experiencia y de manera más específica, el $21.8 \%$ posee 5 años o menos de experiencia docente. 
Tabla 1

Características sociodemográficas del profesorado participante

\begin{tabular}{lcc}
\hline Edad & Frecuencia & $\%$ \\
\hline $21-30$ & 17 & 21.8 \\
$31-40$ & 20 & 25.6 \\
$41-50$ & 26 & 33.3 \\
$51-60$ & 15 & 19.2 \\
Género & Frecuencia & $\%$ \\
Masculino & 7 & 9 \\
Femenino & 71 & 91 \\
Estudios & Frecuencia & $\%$ \\
NC & 7 & 9 \\
Bachillerato & 0 & 0 \\
Diplomatura/Univers. Medio & 45 & 57.7 \\
Licenciatura/Univers. Superior & 26 & 33.3 \\
Total & 78 & 100 \\
\hline
\end{tabular}

Tabla 2

Características de los centros

\begin{tabular}{lcc}
\hline Tipo de centro & Frecuencia & $\%$ \\
\hline Público & 31 & 39.8 \\
Privado & 15 & 19.2 \\
Concertado & 32 & 41 \\
Total & 78 & 100 \\
\hline
\end{tabular}

Otro de los aspectos tenidos en cuenta es el contacto que se ha establecido con personas con discapacidad. De todos los entrevistados, $53(67.9 \%)$ afirmaron haber tenido o tener contacto con personas con discapacidad; mientras que 16 (20.5\%) dijeron no haberlo tenido; y 9 (11.5\%) no contestaron. Además, entre los que afirman haber mantenido contacto con personas con discapacidad, aluden a razones de índole laboral (33.3\%), familiar $(10.3 \%)$ u ocio/amistad $(6.4 \%)$, contacto que realizan de forma habitual (26.9\%), con personas afectadas de diversas problemáticas (15.4\%).

\section{Instrumento}

Para la recogida de datos, se utilizó la Escala de Actitudes hacia las personas con discapacidad (Verdugo et al., 2002). Se trata de una escala multidimensional com- 
puesta por 37 ítems, que fue desarrollada en España, y que cumple con adecuadas condiciones de fiabilidad y validez. La persona evaluada ha de indicar su grado de conformidad ante las frases que se formulan, tanto de forma positiva como negativa, a través de las siguientes puntuaciones: Estoy muy de acuerdo (MA) (1); Estoy bastante de acuerdo (BA) (2); Estoy parcialmente de acuerdo (PA) (3); Estoy parcialmente en desacuerdo (PD) (4); Estoy bastante en desacuerdo (BD) (5); Estoy en total desacuerdo (TD) (6). Una menor puntuación indica una actitud más favorable. En esta escala, se pueden encontrar cinco factores diferentes. El primer factor, Valoración de las capacidades y limitaciones (que engloba los ítems número 1, 2, 4, 7, 8, $13,16,21,29$ y 36), hace referencia a la concepción de las personas con discapacidad que tiene la persona evaluada. El segundo, Reconocimiento/Negación de derechos (que recoge los ítems 6,9 , 12, 14, 15, 17, 22, 23, 27, 35 y 37), alude al reconocimiento de los derechos fundamentales que han de tener las personas con discapacidad. El tercer factor, Implicación personal (incluye los ítems número 3, 5, 10, 11, 25, 26 y 31), refleja la interacción que tendría la persona evaluada con las personas con discapacidad. En el caso del factor cuarto, Calificación genérica, agrupa los ítems número 18, 20, 24,28 y 34 y revela las atribuciones que se realizan acerca de la personalidad y conducta de las personas con discapacidad. El quinto factor, Asunción de roles, que hace referencia a las creencias que la persona evaluada tiene acerca de la concepción que tienen las personas con discapacidad de sí mismos, aglutina los ítems 19, 30, 32 y 33. Además, este cuestionario recoge información general acerca de la edad, el sexo, los estudios y la profesión de las personas encuestadas. Por otro lado, también existen ítems relacionados con el contacto o no con personas con discapacidad, debiendo especificar, en caso afirmativo, la razón del contacto (familiar, laboral, asistencial, ocio/amistad, otras razones), la frecuencia (casi permanente, habitual, frecuente o esporádica) y el tipo de discapacidad de la persona con la que se tiene contacto (física, auditiva, visual, intelectual o múltiple).

\section{Procedimiento}

Para llevar a cabo esta investigación, se seleccionaron 14 centros de la provincia de Granada en los que se impartiera Educación Infantil, incluyendo en la muestra tanto centros del primer y segundo ciclo, como centros públicos, privados y concertados.

Una vez escogidos los centros, a través de una carta enviada por correo electrónico al centro, explicando la relevancia de la investigación, se solicitaba la colaboración de los maestros y maestras de Educación Infantil. A aquellos docentes que estuvieran dispuestos a participar, se les proporcionó el cuestionario con el fin de contestarlo de forma anónima y voluntaria. También se transcribió a ordenador con el fin de facilitar la participación de aquellos centros que se encuentran más lejos, sin necesidad de trasladarse. Posteriormente, se procedió a la recogida en los centros del instrumento ya cumplimentado, que se llevó a cabo de una forma escalonada, según iban informando de la realización de este. Una vez llevada a cabo la recolección, se pudo comprobar que ha existido un alto porcentaje de participación (78\%) por parte de los docentes que se seleccionaron en la primera fase de la investigación. 
Las respuestas recogidas en dicho cuestionario se recogieron en una base de datos, y se analizaron mediante el paquete estadístico SPSS, en su versión 22. Gracias a esto, se pudieron extraer los resultados y conclusiones que se expondrán posteriormente.

\section{Análisis de datos}

Se realizaron diferentes análisis estadísticos para alcanzar el objetivo de estudio. En primer lugar, un análisis descriptivo de los datos, seguido de diferentes ANOVAS univariados.

\section{Resultados}

Para llevar a cabo el análisis, fue necesario codificar de forma inversa, en cuanto a los valores que le asigna la escala, los ítems que expresan una valoración negativa (1, $2,4,5,6,7,8,10,15,17,18,23,24,25,26,28,29,31,34,35$ y 37).Para realizar el análisis de los resultados obtenidos a través de los cuestionarios, nos planteamos un análisis descriptivo. En la tabla 3 se presentan los estadísticos descriptivos que componen los cinco factores de la escala. En general, podemos observar que la actitud que presentan los docentes de infantil encuestados es bastante positiva, existiendo pequeñas diferencias entre los distintos factores.

Tabla 3

Estadísticos descriptivos por factores

\begin{tabular}{lcc}
\hline & M & DT \\
\hline Media Factor I & 2.07 & .77 \\
Media Factor II & 1.66 & .58 \\
Media Factor III & 1.33 & .54 \\
Media Factor IV & 2.17 & .81 \\
Media Factor V & 2.28 & 1.03 \\
Media Global & 2.27 & 1.03 \\
\hline
\end{tabular}

Factor I. Valoración de capacidades y limitaciones: entre los resultados obtenidos, referentes a la concepción que se tiene de las personas con discapacidad, podemos destacar el rechazo de la gran mayoría de los encuestados a la idea de que de las personas con discapacidad no se puede esperar mucho $(\mathrm{M}=1.38 ; \mathrm{DT}=.99)$, confiando, por tanto, en su competencia en el ámbito laboral $(\mathrm{M}=1.36$; $\mathrm{DT}=.83)$. Sin embargo, para una parte importante de los entrevistados, las personas con discapacidad funcionan en muchos aspectos como niños ( $\mathrm{M}=2.85$; DT=1.46).

Factor II. Reconocimiento/Negación de derechos: en este factor, los resultados reflejan unas actitudes bastante positivas en general. Los docentes de Educación 
Infantil entrevistados reflejan especialmente su desacuerdo en cuanto a la idea de que las personas con discapacidad deberían vivir con personas afectadas con el mismo problema $(\mathrm{M}=1.22 ; \mathrm{DT}=.59)$. De la misma forma, reconocen sus derechos básicos, como la posibilidad de poder casarse $(\mathrm{M}=1.73$; $\mathrm{DT}=1.01)$, de poder divertirse $(\mathrm{M}=1.44$; $\mathrm{DT}=1.27)$ e incluso tener las mismas posibilidades laborales que los demás (M=1.88; $\mathrm{DT}=1.26)$.

Factor III. Implicación personal: en este factor, en el cual se refleja la interacción de la persona encuestada con las personas con discapacidad, todos los encuestados están concienciados de la necesidad de no excluir de la sociedad a las personas con discapacidad $(\mathrm{M}=1.04 ; \mathrm{DT}=.29)$. De la misma forma, estarían dispuestos a trabajar con personas con discapacidad $(\mathrm{M}=1.28$; $\mathrm{DT}=.69)$, y permitirían a su hijo acudir al cumpleaños de un niño con NEE (M=1.18; DT= .95).

Factor IV. Calificación genérica: la mayor parte de los encuestados opinan que las personas con discapacidad son sociables $(\mathrm{M}=2.00$; $\mathrm{DT}=1.05)$, pero sin embargo, también existe una cierta tendencia a pensar que las personas con discapacidad son generalmente suspicaces $(\mathrm{M}=2.86$; $\mathrm{DT}=1.47)$.

Factor V. Asunción de roles: los resultados obtenidos reflejan la creencia de los maestros de que las personas con discapacidad pueden tener una vida social normal $(\mathrm{M}=2.05 ; \mathrm{DT}=1.45)$. Sin embargo, no están tan de acuerdo con la afirmación de que las personas con discapacidad confían en sí mismas tanto como el resto $(\mathrm{M}=2.42 ; \mathrm{DT}=1.32)$.

Después de realizar el análisis descriptivo de los diferentes factores que componen la escala, se realizó un análisis multivariado de la varianza (ANOVA) para analizar los efectos que la variables género, edad, años de experiencia docente, contacto y formación-nivel educativo, tienen sobre los diferentes factores que componen la escala de actitudes hacia la discapacidad (Factor I. Valoración de las capacidades y limitaciones, Factor II. Reconocimiento/Negación de derechos; Factor III. Implicación personal; Factor IV. Calificación genérica; Factor V. Asunción de roles), mostrando igualmente las medias y desviaciones típicas por titulación. Se encuentran diferencias significativas en cuanto al nivel educativo-formación entre los dos grupos, docentes de primer y de segundo ciclo de educación infantil. Los docentes que trabajan en primer ciclo de educación infantil (0-3 años), cuentan generalmente con la formación correspondiente a un ciclo formativo de grado superior (Técnico Superior en Educación Infantil), sin embargo para la docencia en el segundo ciclo de educación infantil (3-6 años), se requeriría formación universitaria (Grado de Educación Infantil). Así se observa que los docentes que imparten docencia en segundo ciclo de educación infantil, muestran en general actitudes más positivas hacia la discapacidad. Es el grupo con actitudes más favorables en el Factor IV (Calificación genérica), F (1.76) $=7.221, \mathrm{p}<.047$.

No se encontraron diferencias significativas en los diferentes factores de la escala, en relación a las variables género $\mathrm{F}(1.76)=.023$, $\mathrm{p}<.881$; edad $\mathrm{F}(3.74)=.260, \mathrm{p}<.854$, años de experiencia docente, $\mathrm{F}(9.68)=1.371, \mathrm{p}<.219$; $\mathrm{y}$ contacto con personas discapacitadas, $\mathrm{F}(2.75)=1.751, \mathrm{p}<.181$. 
Tabla 4

Estadísticos descriptivos nivel educativo-formación

\begin{tabular}{|c|c|c|c|c|c|c|}
\hline & Formación & $M$ & DT & ET & $\mathrm{F}$ & $\mathrm{P}$ \\
\hline \multirow{2}{*}{ Factor II } & $1^{\circ}$ ciclo & 2.17 & .72 & .013 & .654 & .421 \\
\hline & $2^{\underline{o}}$ ciclo & 2.02 & .81 & .011 & & \\
\hline \multirow{2}{*}{ Factor II } & $1^{\mathrm{o}}$ ciclo & 1.71 & .50 & .096 & .354 & .554 \\
\hline & $2^{\underline{o}}$ ciclo & 1.63 & .63 & .089 & & \\
\hline \multirow{2}{*}{ Factor III } & $1^{\mathrm{o}}$ ciclo & 1.29 & .45 & .085 & .133 & .717 \\
\hline & $2^{o}$ ciclo & 1.34 & .59 & .083 & & \\
\hline \multirow{2}{*}{ Factor IV } & $1^{\mathrm{o}}$ ciclo & 2.49 & .72 & .013 & 7.221 & .047 \\
\hline & $2^{\underline{o}}$ ciclo & 1.99 & .81 & .0115 & & \\
\hline \multirow{2}{*}{ Factor V } & $1^{\mathrm{o}}$ ciclo & 2.26 & 1.00 & .189 & .005 & .945 \\
\hline & $2^{\underline{o}}$ ciclo & 2.28 & .06 & .150 & & \\
\hline
\end{tabular}

${ }^{*} p<.005$

\section{Discusión y conclusiones}

De acuerdo con los datos obtenidos, se podría afirmar que existe una actitud positiva generalizada en los docentes, tanto del primer como del segundo ciclo, de Educación Infantil de Granada. Similares resultados podemos encontrarnos en otras investigaciones llevadas a cabo con docentes de esta misma etapa (Gal et al., 2010; Santiago \& Marrero, 2003), y en etapas superiores (Álvarez et al., 2005; Comes et al., 2011; Doménech et al., 2004; Martínez \& Bilbao, 2011; Mayo, 2012; Rodríguez-Martín \& Álvarez-Arregui, 2015; Sánchez, 2011). La cuestión es qué factores contribuyen a experiencias positivas de algunos docentes, y negativas de otros. Se sugiere la importancia de indagar en este aspecto, así como llevar a cabo estudios de carácter longitudinales. En consonancia con la revisión bibliográfica utilizada, en segundo lugar se indagó en la influencia de variables tales como género, edad, años de experiencia docente, contacto y formación-nivel educativo del docente. No se encontraron diferencias significativas en las variables, género, edad y contacto con personas discapacitadas. En relación al género de los participantes, los datos obtenidos al ser inconsistentes, están en la dirección de otros autores (Galović et al., 2015; García \& Alonso, 1985; Parasuram, 2006; Sánchez, 2011). Sin embargo, e incidiendo en esta variable, y como una limitación del estudio, apuntamos al tamaño y la paridad en la muestra. Por tanto, para futuras investigaciones se considerará esencial atender a esta limitación. A pesar de ello, sabemos que es una tarea difícil ya que como demuestra Ramos (2002), la carrera de Educación Infantil presenta una elevada presencia femenina en sus aulas, constituyendo el $90 \%$ de los alumnos.

Otras variables analizadas, la edad y los años de experiencia docente, no parecen influir en el presente estudio en las actitudes de los docentes hacia las personas con discapacidad, a pesar de que otros autores apuntan a que los docentes más jóvenes (Dengra et al., 1991; García \& Alonso, 1985), y por tanto con menos años de experiencia docente (Parasuram, 2006) pudieran tener actitudes más positivas. No obstante, hay 
que considerar que la mayor parte de los participantes del estudio tienen una edad superior a 51 años, lo que puede ser un factor explicativo. Además, en el estudio otra variable que parece no influir en las actitudes de los docentes de infantil es el haber mantenido contacto con personas discapacitadas, lo que no concuerda con otros estudios en los que se pone de manifiesto la importancia de esta variable (Bilbao, 2008; Díaz, 2004; Kwon et al., 2017; Martínez \& Bilbao, 2011), por lo que se apunta a la necesidad de indagar sobre la frecuencia del contacto con personas discapacitadas, pues existe cierta controversia respecto a la influencia que la frecuencia del contacto ejerce sobre las actitudes manifestadas. Diferentes autores (Gómez \& Infante, 2004; Moreno et al., 2006; Parasuram, 2006; Yazbeck et al., 2004) señalan que esta variable no parece tener peso en el desarrollo de actitudes positivas hacia las personas con discapacidad. Otros apuntan a que un contacto esporádico ejercería una influencia positiva (García \& Hernández, 2011; López-Ramos, 2004), frente a un contacto más frecuente, habitual permanente que daría lugar a actitudes más desfavorables (Muratori et al., 2010).

Por último, en el estudio encontramos como el nivel educativo-formación, influye sobre las actitudes de los docentes, siendo los de segundo ciclo, por tanto los que contarían con estudios universitarios, y mayor formación, los que tienen actitudes más positivas. Diferentes trabajos han puesto de manifiesto que la información sobre la discapacidad recibida a lo largo de su formación universitaria podría ser uno de los determinantes de sus actitudes (Carberry et al.,1981) comprobándose además que un mayor nivel de información y formación se relaciona con actitudes más positivas (Verdugo et al., 2002).

Nos encontramos ante un estudio pionero en su campo, ya que no se han encontrado investigaciones que aborden ambos ciclos de Educación Infantil. Esto nos permite comparar y analizar si los docentes de esta etapa tienen la formación necesaria para llevar a cabo una educación de calidad para todo el alumnado, sin ningún tipo de exclusión. La necesidad de centrarnos en esta etapa educativa radica en el carácter preventivo de la misma, en la medida en que la inclusión educativa como prevención para la exclusión social es el medio más efectivo para combatir las actitudes de discriminación (Echeita, 2006). De la misma forma, las actitudes de profesores hacia la inclusión de sus estudiantes, es un potente predictor de la calidad de la educación para la inclusión de estudiantes con discapacidad (Cook, Tankersley, Cook \& Landrum, 2000).

\section{Referencias}

Abós, P., \& Polaino, A. (1986). Integración de deficientes mentales educables: Un estudio de actitudes docentes. Revista Española de Pedagogía, 44(172), 193-206.

Alaminos, A. (2006). El muestreo en la investigación social. En A. Alaminos \& J. L. Castejón (Ed.), Elaboración, análisis e interpretación de encuestas, cuestionarios y escalas de opinión (pp. 41-67). Alicante, España: Vicerrectorado de Calidad y Armonización Europea, Instituto de Ciencias de la Educación.

Álvarez, M., Castro, P., Campo-Mon, M.A., \& Álvarez-Martino, E. (2005). Actitudes de los maestros ante las necesidades educativas específicas. Psicothema, 17(4), 601-606. Recuperado de http://www.psicothema.es/pdf/3152.pdf 
Ashman, A.F. (1982). Prevention or cure? Changing Attitudes toward Retarded persons. Mental retardation Bulletin, 10(1), 13-85.

Bilbao, M.C. (2008). La integración de personas con discapacidad en la educación superior. Percepciones y demandas de docentes y estudiantes en la Universidad de Burgos. (Tesis Doctoral). Facultad de Humanidades y Educación. Universidad de Burgos.

Carberry, H., Waxman, B., \& Mckain, D. (1981). An in-service workshop model for class teachers concerning mainstreaming of the learning disabled child.Journal of LearningDisabilities, 14(1), 26-28.

Chiner, E. (2011). Las percepciones y actitudes del profesorado hacia la inclusión del alumnado con necesidades educativas especiales como indicadores del uso de prácticas educativas en el aula. (Tesis doctoral). Universidad de Alicante, España.. Recuperado de http:// rua.ua.es/dspace/handle/10045/19467

Clark, B.A. (1976). Teacher attitudes toward integration of children with handicaps. Education and training of the Mentally Retarded, 11(4), 333-335.

Comes, G., Parera, B., Vedriel, G., \& Vives, M. (2011).La inclusión del alumnado con discapacidad en la Universidad: la opinión del profesorado. Innovación educativa, 21,173-183. Recuperado de http://www.usc.es/revistas/index.php/ie/ article/ viewFile/32/149

Cook, B.G., Tankersley, M., Cook, L., \& Landrum, T. J. (2000).Teachers' attitudes toward their included students with disabilities.Exceptional Children, 67(1), 115-135.

Cortés, M.C. (2010). La inclusión educativa del alumnado: ventajas e inconvenientes. Innovación y experiencias educativas, 27, 1-8. Recuperado de https://archivos.csif.es /archivos/andalucia/ensenanza/revistas/csicsif/revista/pdf/Numero_27/MARIA_ CRISTINA_CORTES_ARIZA_02.pdf

Dengra, R., Durán, R., \& Verdugo, M.A. (1991). Estudio de las variables que afectan a las actitudes de los maestros hacia la integración escolar de niños con necesidades especiales. Anuario Español e Iberoamericano de Investigación en Educación Especial, 47-88.

Díaz, G. (2004). Los estudiantes con discapacidad en la Universidad de Las Palmas de Gran Canaria. Las Palmas de Gran Canaria: Servicio de Publicaciones de la Universidad.

Doménech, V., Esbrí, J. V., González, H. A., \& Miret, L. (2004). Actitudes del profesorado hacia el alumnado con necesidades educativas especiales derivadas de discapacidad. Comunicación presentada a Novenes Jornades de Foment de la Investigació, Castelló.

Echeita, G. (2006). Educación para la inclusión o educación sin inclusiones. Madrid: Narcea. Eichinger, J., Rizzo, T., \& Sirotnik, B. (1991).Changing attitudes toward people with disabilities. Teacher Education y SpecialEducation,14, 121-126.

Escámez, J., García, R., Pérez, C., \& Llopis, A. (2007). El aprendizaje de valores y actitudes. Teoría y práctica. Madrid: Octaedro/OEI.

Gal, E., Schreur, N., \& Engel-Yeger, B. (2010). Inclusion of children with disabilities: Teacher's Attitudes and requirements for environmental accomodations. International Journal of Special Education, 25(2), 89-99. Recuperado de https://files. eric.ed.gov/fulltext/EJ890588.pdf 
Galović, D., Brojčin, B., \& Glumbić, N. (2015). The attitudes of teachers toward inclusive education in Vojvodina. International Journal of Inclusive Education, 18(12), 1262-1282. doi: 10.1080/13603116.2014.886307.

Garabal-Barbeira, J. (2015). Actitudes de docentes y estudiantes hacia la discapacidad en la Universida de da Coruña. Revista de Estudios e Investigación en Psicología y Educación, 11, 11-19. doi: 10.17979/reipe.2015.0.11.220

García, J.N., \& Alonso, J.C. (1985). Actitudes de los maestros hacia la integración escolar de niños con necesidades especiales. Infancia y Aprendizaje, 30, 51-68. Recuperado de http://dialnet.unirioja.es/servlet/articulo? codigo $=667387$

García, G.A., \& Hernández, S. (2011). Actitudes hacia la discapacidad de jóvenes y adultos de Chiapas. Universitas Psychologica, 10(3), 817-827. Recuperado de http:// revistas.javeriana.edu.co/index.php/revPsycho/article/viewFile/594/1174

Gómez, V., \& Infante, M. (2004). Actitudes de los estudiantes de educación hacia la integración de las personas con discapacidad y hacia la educación multicultural. Cultura y Educación, 16, 371-383. Recuperado de https://www.researchgate.net/ publication/233657610_Actitudes_de_los_estudiantes_de_educacion_hacia_la_ integracion_de_personas_con_discapacidad_y_hacia_la_educacion_multicultural_ Education_student's_attitudes_towards_integrating_people_with_disabilitie

Harasymiw, S. J., \& Horme, M.D. (1976). Teacher attitudes toward handicapped children and regular class integration. The Journal of special Education, 10(4), 393-400.

Kwon, K., Hong, S.Y., \& Jeon, H.J. (2017). Classroom Readiness for Successful Inclusion: Teacher Factors and Preschool Children's Experience With and Attitudes Toward Peers With Disabilities. Journal of Research in Childhood Education, 31(3), 360-378. Recuperado de http://dx.doi.org/10.1080 /02568543.2017.1309480

León, M.J. (1995). Las actitudes del profesor tutor de alumnos con necesidades educativas especiales en su aula acerca de la integración escolar. Una revisión de las investigaciones de campo. Revista de Educación de la Universidad de Granada, 8, 141-152.

López-Ramos, V. M. (2004). Estudio sobre las actitudes hacia las personas con discapacidad entre los estudiantes de la Universidad de Extremadura. (Tesis doctoral no publicada). Universidad de Extremadura, España.

Martínez, M.A., \& Bilbao, M.C. (2011). Los docentes de la universidad de Burgos y su actitud hacia las personas con discapacidad. Revista Española sobre Discapacidad Intelectual, 42(4), 50-78. Recuperado de http://sid.usal.es/idocs/F8/ ART19441/240-8\%20Mart\%C3\%ADnez.pdf

Mayo, M.E. (2012). La atención a la diversidad en las aulas universitarias: necesidades y dificultades del personal docente e investigador. Recuperado de: http://diversidad. murciaeduca.es/publicaciones/dea2012/docs/emayo2.pdf

Ministerio de Educación, Cultura y Deporte (2001). Education Development. Madrid: Secretaría General Técnica. Subdirección General de Información y Publicaciones.

Moreno, F. J., Rodríguez, I. R., Saldaña, D., \& Aguilera, A. (2006). Actitudes ante la discapacidad en el alumnado universitario matriculado en materias afines. Revista Iberoamericana de Educación, 40, 1-12. Recuperado de http://riberdis.cedd. net/ bitstream/handle/11181/4373/Actitudes_ante\%20_la_discapacidad_en_ el_alumnado_universitario_matriculado_en_materias_afines.pdf?sequence=1 \&rd=0031924102003194 
Muratori, M., Guntín, C., \& Delfino, G. (2010). Actitudes de los adolescentes hacia las personas con discapacidad: un estudio con alumnos del polimodal en la zona norte del conurbano bonaerense [en línea]. Revista de Psicología, 6(12), 39-56. Recuperado de http://bibliotecadigital.uca.edu.ar/repositorio/revistas/actitudesadolescentes-hacia-personas-discapacidad.pdf

Noe, R.A. (2002). Employee training and development. New York: McGraw Hill.

Organización Mundial de la Salud. (2011). Informe mundial sobre la discapacidad. Genève: Organización Mundial de la Salud.

Parasuram, K. (2006). Variables that affect teachers' attitudes towards disability and inclusive education in Mumbai, India. DisabilityESociety, 21(3), 231-242. doi:10.1080/09687590600617352

Peralta, F. (2011). Niños diferentes: Los trastornos del desarrollo y su intervención psicopedagógica. Madrid: Ediciones Eunate.

Polo, Ma‥T., Fernández, C., \& Fernández, M. (2017). The Attitudes of Different Partners Involved in Higher Education towards Students with Disabilities. International Journal of Disability Development. doi: 10.1080/1034912X.2017,1406066.

Quinn, M. (1980). Qualitative Evaluation Methods. Beverly Hills, CA: Sage Publications, Inc. Ramos, E. (2002). El alumnado de Magisterio y la elección de la carrera. Apuntes de algunos condicionamientos socioeconómicos y culturales significativos. Campo abierto, 21, 57-74.

Rodríguez-Martín, A., \& Álvarez-Arregui, E. (2015). Universidad y discapacidad. Actitudes del profesorado y de estudiantes. Perfiles educativos, 37(147), 86-102. Recuperado de http://www.redalyc.org/html/132/13233749006/

Rodríguez, E., Etopa, P., \& Rodríguez, A. (2002). Las actitudes de los futuros maestros y maestras de la Educación Infantil hacia la discapacidad: un factor de prevención. El Guiniguada, 11, 171-180. Recuperado de http://ojsspdc.ulpgc.es/ojs/index.php/ ElGuiniguada/article/view/639/566

Sales, A., Moliner, O., \& Sanchiz-Ruiz, M.L. (2001). Actitudes hacia la atención a la diversidad en la formación inicial del profesorado. Revista Electrónica Interuniversitaria de Formación del Profesorado, 4(2), 1-7. Recuperado de https:// dialnet.unirioja.es/servlet/articulo?codigo $=1031317$

Sánchez, A. (2011). La Universidad de Almería ante la integración educativa y social de los estudiantes con discapacidad: Ideas y actitudes del personal docente e investigador. Revista de Educación, 354, 575-603. Recuperado de http://www. revistaeducacion.mec.es/re354/re354_23.pdf

Sánchez, A., \& Carrión, J.J. (2002). Una aproximación a la investigación en educación especial. Revista de Educación, 327, 225-247. Recuperado de https:// www.mecd.gob.es/dctm/revista-de-educacion/articulos327/re3271410520. pdf?documentId=0901e72b812598b5

Santiago, O., \& Marrero, G. (2003). Actitudes hacia las personas con discapacidad en profesionales del primer ciclo de Educación Infantil. Anuario de Filosofía, Psicología y Sociología, 6, 131-158. Recuperado de https://acceda.ulpgc.es:8443/bitstream /10 553/3622/1/0237190_02003_0007.pdf 
Soto, R. (2007). Actitudes docentes de la Universidad de Costa Rica hacia los(as) estudiantes con discapacidad de la Universidad. Revista Educación, 31(1), 11-42. Recuperado de https://revistas.ucr.ac.cr/index.php/educacion/article/view/1251

Stauble, K.R. (2009). Teacher attitudes toward inclusion and the impact of teacher and school variables. University of Louisville, United States - Kentucky.

Tenorio, S. (2011). Formación inicial docente y necesidades educativas especiales. Estudios Pedagógicos, 37(2), 249-265. Recuperado de https://dialnet.unirioja.es/ servlet/articulo? codigo $=4885730$

Toledo, M. (1984). La escuela ordinaria ante el niño con necesidades educativas especiales. Madrid: Santillana.

UNESCO. (1994). Declaración de Salamanca y marco de acción para las necesidades educativas especiales. Salamanca: UNESCO. Recuperado de http://www.unesco.org/education/ pdf/SALAMA_S.PDF

Verdugo, M.A., Jenaro, C., \& Arias, B. (2002). Actitudes sociales y profesionales hacia las personas con discapacidad: estrategias de evaluación e intervención. En M. A. Verdugo (Dir.), Personas con discapacidad. Perspectivas psicopedagógicas y rehabilitadoras (pp. 79-135). Madrid: Siglo XXI.

Yazbeck, M., Mcvilly, K., \& Parmenter, T. (2004). Attitudes Toward People with Intellectual Disabilities. Journal of Disability Policy Studies, 2, 97-112. doi: $10.1177 / 10442073040150020401$

Fecha de recepción: 3 de enero de 2017

Fecha de revisión: 15 de enero de 2017

Fecha de aceptación: 27 de marzo de 2018 
